\title{
Modeling the Effect of Global Warming on the Sustainable Groundwater Management: A Case Study in Bangladesh
}

\author{
ANARUL ISLAM, HAIDER ALI BISWAS \\ Mathematics Discipline, Science Engineering and Technology School \\ Khulna University, Khulna-9208, BANGLADESH
}

\begin{abstract}
The paper deals with a deterministic model for the declination of groundwater level due to deforestation and evaporation that is caused of global warming. The model is governed by three compartments by considering different level of water. The model is analyzed by finding the existence of equilibrium points and also derived the conditions of stability at the equilibrium points by using Jacobian matrix and Routh-Hurwitz criterion for the system of non-linear differential equation. We also observe the qualitative behavior by using phase portrait diagram. Finally, the numerical simulations have been performed to illustrate the effect of deforestation, poor storage of water and evaporation on the groundwater level in support of analytical findings briefly. Our study shows that, groundwater level decreases drastically due to deforestation and global warming.
\end{abstract}

Keywords-Deforestation, Mathematical Modeling, Equilibrium point and Numerical Simulations.

Received: August 2, 2020. Revised: December 3, 2020. Accepted: December 18, 2020. Published: December 29, 2020.

\section{Introduction}

Groundwater is a significance natural resources for public water supply and food production in Bangladesh and all over the world that constitutes 95 percent of the freshwater [3]. About three billion people depend directly upon for drinking water and $40 \%$ of the world's food is produced from irrigated agriculture that relies mainly on groundwater. Moreover, in the rural area, farmers are used freshwater about $70 \%$ for the purpose of cultivation and by 2050 , nine billion people will depend upon an approximated $50 \%$ increase in irrigated agricultural production [15]. Groundwater level declines for many reasons, some of which occurs due to natural phenomena and others are caused by human's activities and it has been declining since the introduction of deep tube wells and shallow tube wells after 1970s. Excessive demand of water, evaporation, pollution, deforestation, poor storage, low rainfall, urbanization, frequent pumping of water are the important causes of groundwater level declination in Bangladesh as well as all over the world. For agricultural use ground water is pumped more frequently, this can be done by fixing a tube well. Most of the industrial waste water are dumped to the water sources. This can be considered another reason for declination of water resources. Due to global warming and change in the climate, huge amounts of surface water and ground water is being evaporated, these also accumulate to the reduction in water resources as well as groundwater resources [17]. Mathematical ecology and modeling have become a well-recognized area of environmental research which is the most enjoyable applications of basic sciences $[15,18]$. The aim of the mathematical biology is to represent a real phenomenon by mathematics and develop a model on the basis of conservation principle of biological processes, exploitation techniques by using the tools of mathematics. Mathematical simulations and modeling play an incredible role for providing quantitative insight into different field of science. It has already contributed to a better understanding of the mechanisms in various field nowadays [4]. Mathematical modeling has gotten attention because modeling 
and simulation of any physical phenomena allows us for rapid assessment. So, it is mainly used to describe the real phenomena which lead to design better prediction, management and control strategies [9].

Groundwater management problem has been discussed by many researchers to their scientific contribution published in international journal. A Mathematical model was proposed and inspected to study the impacts of predicted climate mutation on recharge and groundwater levels [5], whereas the effects of climate change on vegetation is characterized in [15] that is the important factor to store groundwater for long period. Another model based on the causes and quantification was also approached to describe the declination of groundwater level and aquifer dewatering in Dhaka metropolitan area in Bangladesh [7]. However, some articles [2-4, 7, $10-13,15,18-19,22-23]$ are referred for more details in the explanation of global climate change in Bangladesh as well as all over the world, interactions among atmospheric, surface and groundwater with the significance factors for depletion of ground layer and development of mathematical model on groundwater level declination. In this paper, we would like to propose a mathematical model on groundwater management. The aim of this study is to describe the impacts of climate change in the environment like deforestation, pollution, evaporation and frequent pumping of water on groundwater level. We develop a mathematical model and study the existence and stability of the model with nonlinear Lyapunov function. Lastly, numerical simulations will be performed to show the effectiveness of the analytical analysis of groundwater model.

\section{Present Situation of Groundwater Declination in Bangladesh}

Groundwater is an imperishable, renewable and sustainable natural resource in Bangladesh. In the wet or monsoon season (June-September), groundwater sustains it's dynamism by recharging, through rainfall and flooding and seceding during the dull season (March-May) due to withdrawal for various application, especially for cultivation. Agricultural yield of Bangladesh has successively increased over the last few decades while we have reasons to be happy about to fact that we must ensure this is not just a fleeting surge but a sustainable propensity. Groundwater level were near or very close to the ground surface in some places after the monsoon period and at the end of the dry season, groundwater tables declined due to evaporation, evapotranspiration and inter-basin flow out of the aquifers. The present status of groundwater level in Bangladesh is given from 2000 to 2018 in Figure 1.

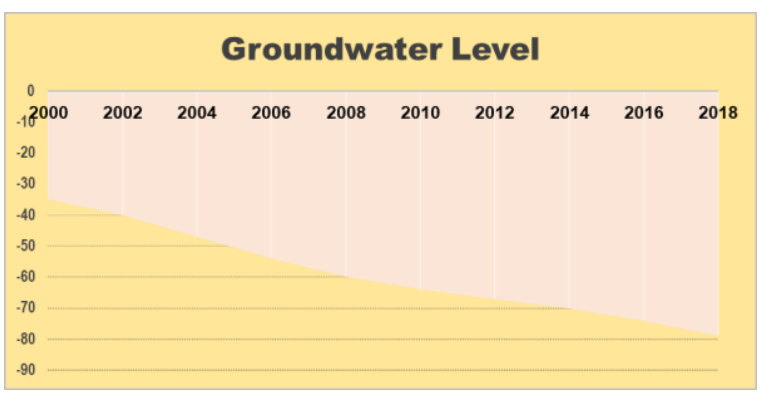

Figure 1. Declination of groundwater level during dry season since 2000 to 2018 in Bangladesh [3].

\section{Mathematical Formulation of the Model}

To construct a mathematical model on groundwater level, firstly we have to know the biological interactions and background among the state variables (i.e. atmosphere, surface and ground water).

According to hydrologic cycle, water vaporizes to become the part of the atmosphere from the oceans and the land surface; then water vapor is deported and lifted in the atmosphere until it condenses and precipitates on the surface or the oceans. Through plantation and vegetation precipitated water can be intercepted for overland flow on the ground surface. Water then infiltrates into the ground as subsurface flow and discharge into streams as surface runoff. Finally, the intercepted water and surface runoff returns again to the atmosphere through evaporation directly from the soil and vegetation surface or transpired from plant leaves. In this way hydrologic cycle continues. The interaction of 
atmosphere, surface and ground water is shown in the Figure 2.

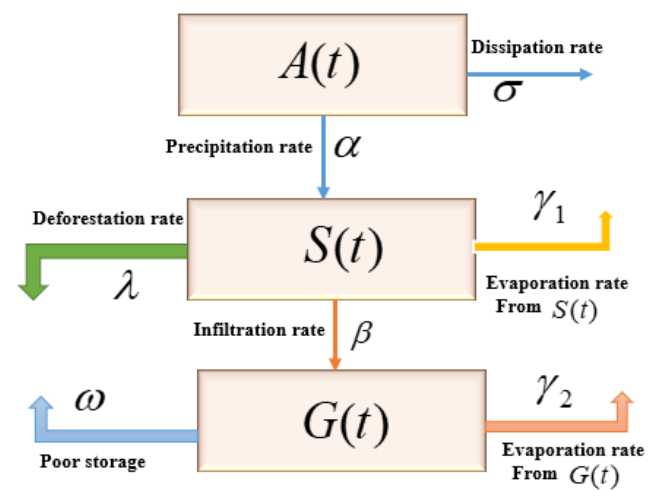

Figure 2: The schematic diagram with biological interactions of groundwater model.

Considering the schematic diagram in Figure 2, we formulate the model with the following system of nonlinear differential equations:

$\frac{d A}{d t}=\gamma_{1} S(t)+\gamma_{2} G(t)-\alpha A(t)-\sigma A(t)$

$\frac{d S}{d t}=\alpha A(t)-\beta G(t) S(t)-\gamma_{1} S(t)+\omega G(t)$

$\frac{d G}{d t}=\beta G(t) S(t)-\gamma_{2} G(t)-\omega G(t)-\lambda G(t)$

with $\quad$ initial
$A(0)=A_{0}, S(0)=\mathrm{S}_{0}$ and $G(0)=G_{0}$

and the total amount of water, $N(t)=A(t)+S(t)+G(t)$.

Here, $A(t), S(t)$ and $G(t)$ are the three state variables that represent the levels of atmospheric water, surface water and groundwater respectively at time $t$.

In the model (1)-(3), we have considered the parameter $\alpha$ as precipitation rate from atmosphere to surface water and $\sigma$ is the dissipation rate of atmospheric water. The infiltration rate from surface to groundwater be $\beta$ whereas $\gamma_{1}$ and $\gamma_{2}$ are the evaporation rate from surface and groundwater to atmospheric water respectively. Moreover $\lambda$ and $\omega$ be the deforestation rate and rate of poor storage system from ground level.

\section{Qualitative Analysis of the Model}

We have to analyze the qualitative behavior of the solutions in the neighborhood of the equilibrium points.

\section{A. Boundedness of the Model}

To prove the system mathematically and biologically well posed, the following lemma has to be satisfied.

Theorem 1: The region $\Omega=\left\{(A(t), S(t), G(t)) \in \square_{+}^{3}: 0 \leq N \leq \frac{\eta}{\sigma+\lambda}\right\} \quad$ is a positively invariant set for the model (1)-(3).

Proof: Since the total amount of water is $N(t)$, then $N(t)=A(t)+S(t)+G(t)$.

The rate of change of total amount of water is

$\frac{d N}{d t}=\frac{d A}{d t}+\frac{d S}{d t}+\frac{d G}{d t}$

$$
=-\sigma N-\lambda(N-A-S)
$$

After simplifying, we get the differential inequality as

$$
\Rightarrow \frac{d N}{d t}+(\sigma+\lambda) N \leq \eta
$$

(4)

By solving the differential equation (4), we obtain

$$
N(t) \leq \frac{\eta}{\sigma+\lambda}+\left(N_{0}-\frac{\eta}{\sigma+\lambda}\right) e^{-(\sigma+\lambda)}
$$

Taking limit as $t \rightarrow \infty$, we get $0<N(t) \leq \frac{\eta}{\sigma+\lambda}$

Thus, we conclude that the region $\Omega$ is the positively invariant set induced by the model (1)-(3) [16]. Therefore, the model is both mathematically and biologically well-posed in the region $\Omega$.

Hence, the Theorem 1 is proved.

\section{B. Positivity of the Solution of the Model}

Theorem 2: If $A(0)>0, S(0)>0$ and $G(0)>0$

then the solutions $A(t), S(t), G(t)$ of the model (1) are all positive [5]. 
Proof: To prove the Theorem 2, we will use the system of differential equations (1)-(3).

The first equation of the model (1)-(3), can be written as $\frac{d A}{d t} \geq-(\alpha+\sigma) A$

$$
\Rightarrow \frac{d A}{d t}+(\alpha+\sigma) A \geq 0
$$

By solving (6), we get

$$
A(t) \geq c_{1} e^{-(\alpha+\sigma) t}
$$

where $c_{1}$ is an integrating constant

To find the value of constant $c_{1}$ we apply the initial condition at $t=0, A(0)=A_{0}$. we get $A_{0} \geq c_{1}$

$$
A(t) \geq A_{0} e^{-(\alpha+\sigma) t} .
$$

Therefore $A(t)>0$ for all $t \geq 0$.

Similarly, we obtain from the differential equations (2) and (3) that, $S(t) \geq 0$, and $G(t) \geq 0$ for all $t \geq 0$.

Hence the Theorem 2 is proved.

\section{Existence of the equilibrium}

To find the equilibrium points of the model (1)(3), we have to solve

$$
\frac{d A}{d t}=\frac{d S}{d t}=\frac{d G}{d t}=0
$$

For pollution free and without pumping from groundwater, we consider $G=G_{0}$ (i.e. initial value of $G(t))$. Let $\bar{E}_{1}(\bar{A}, \bar{S}, \bar{G})$ be the pollution free equilibrium point. Using $G=G_{0}$ in the system (9), and solving the system of algebraic equation, we get

$$
\bar{E}_{1}(\bar{A}, \bar{S}, \bar{G})=\left(\frac{\gamma_{1} S+\gamma_{2} G_{0}}{\alpha+\sigma}, \frac{\gamma_{2}+\omega+\lambda}{\beta}, G_{0}\right)
$$

Again, let $E_{2}^{*}\left(A^{*}, S^{*}, G^{*}\right)$ be the equilibrium point of the model (1)-(3) when all the activities are performed in the groundwater (i.e. the general equilibrium point), then we obtain

$$
E_{2}^{*}\left(A^{*}, S^{*}, G^{*}\right)=\left(\begin{array}{c}
\frac{\gamma_{1} S^{*}+\gamma_{2} G^{*}}{\alpha+\sigma}, \frac{\gamma_{2}+\omega+\lambda}{\beta}, \\
\frac{S^{*}\left[\alpha \gamma_{1}-\gamma_{1}(\alpha+\sigma)\right]}{\left(\beta S^{*}+\omega\right)(\alpha+\sigma)-\alpha \gamma_{2}}
\end{array}\right)
$$

where, $A^{*}=\frac{\alpha \lambda^{2} \gamma_{1}+\alpha \lambda \gamma_{1} \gamma_{2}+\alpha \lambda \gamma_{1} \omega}{\alpha \beta\left(\alpha \lambda+\lambda \sigma+\gamma_{2} \mu\right)}$,

$$
\begin{aligned}
& S^{*}=\frac{\lambda+\gamma_{2}+\omega}{\beta} \text { and } \\
& G^{*}=\frac{\lambda \gamma_{1} \sigma+\gamma_{1} \gamma_{2} \sigma+\gamma_{1} \sigma \omega}{\left(\alpha \lambda+\sigma \lambda+\gamma_{2} \sigma-\alpha \beta \lambda-\beta \sigma \lambda-\beta \gamma_{2} \sigma\right)}
\end{aligned}
$$

$$
\text { D. Stability at } \bar{E}_{1}(\bar{A}, \bar{S}, \bar{G})
$$

Theorem 3: The equilibrium point $\bar{E}_{1}(\bar{A}, \bar{S}, \bar{G})$ of the model (1)-(3) is asymptotically stable if $a_{1}>0, a_{3}>0$ and $a_{1} a_{2}>a_{3}$, otherwise it is unstable [30, 48].

Proof: The characteristic equation of the system is given as

$$
|J-\lambda I|=0
$$

After simplifying, we get

$$
\begin{aligned}
& \left(A_{1}-\lambda\right)\left(A_{2}-\lambda\right)\left(A_{3}-\lambda\right)-\left(A_{1}-\lambda\right) A_{4} A_{5}- \\
& A_{6}\left(A_{3}-\lambda\right)-A_{7} A_{4}=0 \\
& \text { (10) } \\
& \text { where, } A_{1}=-\alpha-\sigma, A_{2}=-\beta G_{0}-\gamma_{1}, \\
& A_{3}=\beta \bar{S}-\gamma_{2}-\omega-\lambda, A_{4}=\beta G_{0}-G_{0}, \\
& \qquad A_{5}=-\beta \bar{S}+\bar{S}+\omega \text {, and } A_{6}=\alpha \gamma_{1}, A_{7}=\alpha \gamma_{2}
\end{aligned}
$$

After simplifying the equation (10), we obtain

$\Rightarrow \lambda^{3}+a_{1} \lambda^{2}+a_{2} \lambda+a_{3}=0$

where, $a_{1}=\left\{-\left(A_{1}+A_{2}+A_{3}\right)\right\}$,

$a_{2}=\left\{A_{1}\left(A_{2}+A_{3}\right) A_{2} A_{3}-A_{4} A_{5}-A_{6}\right\}$, and

$a_{3}=A_{1} A_{4} A_{5}-A_{1} A_{2} A_{3}+A_{3} A_{6}-A_{4} A_{7}$.

Here, $a_{1}=\left\{-\left(A_{1}+A_{2}+A_{3}\right)\right\}>0$, similarly, $a_{3}>0$ and $a_{1} a_{2}>a_{3}$.

From the Routh-Hurwitz criterion [14], we know that all the eigen values of (11) have negative real roots if and only if $a_{1}>0, a_{3}>0$ and $a_{1} a_{2}>a_{3}$. 
Hence, the equilibrium point $\bar{E}_{1}(\bar{A}, \bar{S}, \bar{G})$ of the model (1)-(3) is asymptotically stable for $a_{1}>0, a_{3}>0$ and $a_{1} a_{2}>a_{3}$.

\section{E. Stability at $E_{2}^{*}\left(A^{*}, S^{*}, G^{*}\right)$}

Theorem 4: The equilibrium point $E_{2}^{*}\left(A^{*}, S^{*}, G^{*}\right)$ of the model (1)-(3) is asymptotically stable if $b_{1}>0, b_{3}>0$ and $b_{1} b_{2}>b_{3}$, otherwise it is unstable.

Proof: At the equilibrium point $E_{2}^{*}\left(A^{*}, S^{*}, G^{*}\right)$, The characteristic equation for the eigen value $\lambda$ is given as

$$
\begin{gathered}
|J-\lambda I|=0 \\
\Rightarrow\left(B_{1}-\lambda\right)\left(B_{2}-\lambda\right)\left(B_{3}-\lambda\right)-\left(B_{1}-\lambda\right) B_{4} B_{5}- \\
B_{6}\left(B_{3}-\lambda\right)-B_{7} B_{4}=0
\end{gathered}
$$

(12)

where, $B_{1}=-\alpha-\sigma, B_{2}=-\beta G^{*}-\gamma_{1}+G^{*}$,

$B_{3}=\beta S^{*}-\gamma_{2}-\omega-\lambda, B_{4}=\beta G-G$,

$B_{5}=-\beta S^{*}+S^{*}+\omega, \quad B_{6}=\alpha \gamma_{1}$, and $B_{7}=\alpha \gamma_{2}$

After simplifying the equation (12), we get $\lambda^{3}+b_{1} \lambda^{2}+b_{2} \lambda+b_{3}=0$

(13)

From the Routh-Hurwitz criterion, we know that all the eigen values of (13) have negative real roots if and only if $b_{1}>0, b_{3}>0$ and $b_{1} b_{2}>b_{3}$.

So, the equilibrium point $E^{*}\left(A^{*}, S^{*}, G^{*}\right)$ of the model (1)-(3) is asymptotically stable if $b_{1}>0, b_{3}>0$ and $b_{1} b_{2}>b_{3}$, otherwise it is unstable.

\section{Numerical Simulations}

We have performed the numerical simulation by ode45 solver using MATLAB programming language. We use a set of suitable parameter values that is estimated from the real data perspective to Bangladesh [3, 23]. The description of all the parameters with the estimated values used in the simulation is presented in Table 1 . We have considered the initial condition $A_{0}=50 \times 10^{9}, S_{0}=110 \times 10^{9}$ and $G_{0}=60 \times 10^{9}$. Also, we have solved for time interval $t \in[0,20]$ for 20 years. The value of the model parameters is given in Table 1 .

Table 1: Values and Explanation of parameters

\begin{tabular}{|l|l|l|}
\hline \multicolumn{1}{|c|}{ Descriptions } & Symbols & Values \\
\hline Precipitation rate & $\alpha$ & 0.5 \\
\hline Dissipation rate & $\sigma$ & 0.01 \\
\hline Infiltration rate & $\beta$ & 0.3005 \\
\hline Deforestation rate & $\lambda$ & 0.06 \\
\hline Storing rate of water & $\omega$ & 0.02 \\
\hline $\begin{array}{l}\text { Evaporation rate from } \\
\text { surface water }\end{array}$ & $\gamma_{1}$ & 0.09 \\
\hline $\begin{array}{l}\text { Evaporation rate from } \\
\text { ground water level }\end{array}$ & $\gamma_{2}$ & 0.028 \\
\hline
\end{tabular}

We run the program for two state variables, surface water and groundwater level keeping all the values of the parameters same as before. The result obtained in this case is represented in Figure 3.

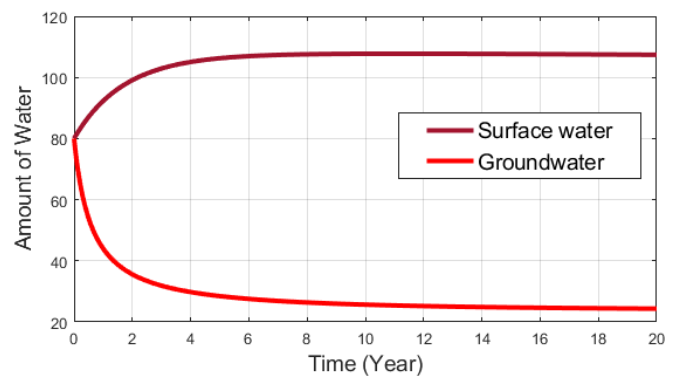

Figure 3. Dynamics of Surface water and Groundwater where Groundwater decrease significantly as a result surface water is increasing day by day.

Figure 3 shows the state trajectories of the two compartments such as surface water and groundwater in the absence of any control measures. We have observed that when no control measure is employed, groundwater level decreases and surface water gradually increases from the initial position. It is occurred due to evaporation of water from the ocean and land surfaces, because this water is temporarily considered as vapor in the atmosphere, and falls back to earth's surface as precipitation, then surface water is formed by the residue of precipitation and melted snow and then it will be stable if no others factor (for examples, drought 
and low streamflow or over streamflow) as employed in the atmosphere.

Now, we solve the model numerically for the case of groundwater to show the change in the groundwater level due to poor storage of water. Taking the values of all parameters into account as in Table 1 and considering the same initial values of the state variables as we did before. The result in this case is presented in Figure 4.

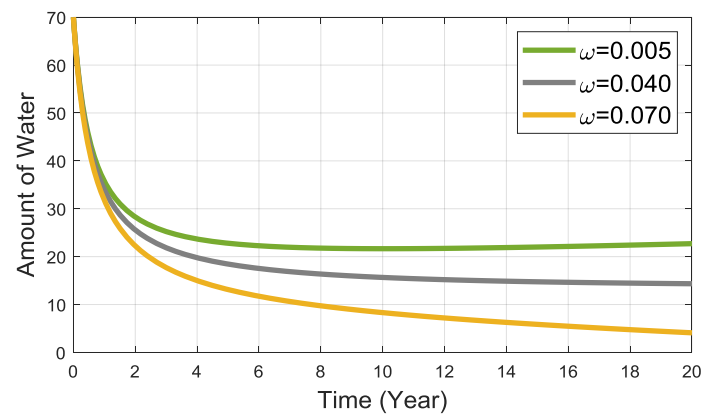

Figure 4. Variation of Groundwater for different values of $\omega$ where falling of groundwater level is significantly increased due to lack of storage system.

Figure 4 represents the variation of groundwater level for different values of $\omega$ with time while pollution and other effects are fixed. We observe that falling of groundwater level is increased tremendously due to lack of storage system.

Next, we have solved the model numerically to show the change in the groundwater level du e to evaporation rate for the parameters in Table. The result in this case is shown in Figure 5.

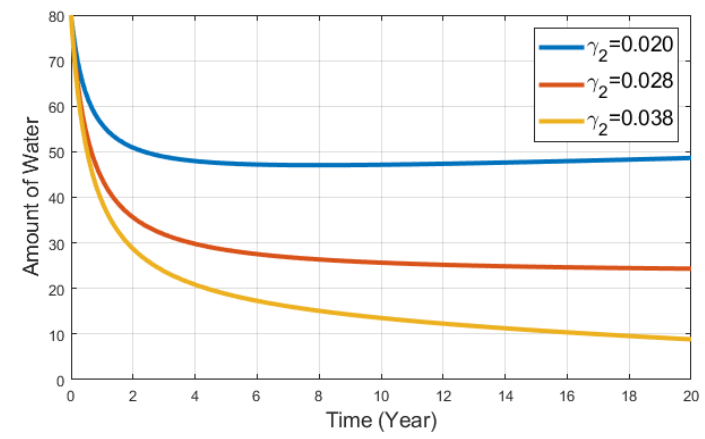

Figure 5. Variation of Groundwater for different values of evaporation rate where falling of groundwater level is extensively increased due to global warming.
In Figure 5, one can see the variation of groundwater level for different values of evaporation rate $\left(\gamma_{2}\right)$ over the time while deforestation rate, pollution rate and other effects are constant. It is easy to understand that falling of groundwater level is increased significantly due to increase of evaporation rate $\gamma_{2}$. That is, falling of groundwater level decreases for the lower value of evaporation rate $\gamma_{2}$ and falling of groundwater level increases for the higher value of evaporation rate $\gamma_{2}$. Global warming can lead to longer periods of droughts, which directly affects availability and dependency on groundwater.

Lastly, we run the program keeping all other values of the parameters same as before and change in the deforestation rate to show the effect of deforestation on groundwater level. The result in this case is presented in Figure 6.

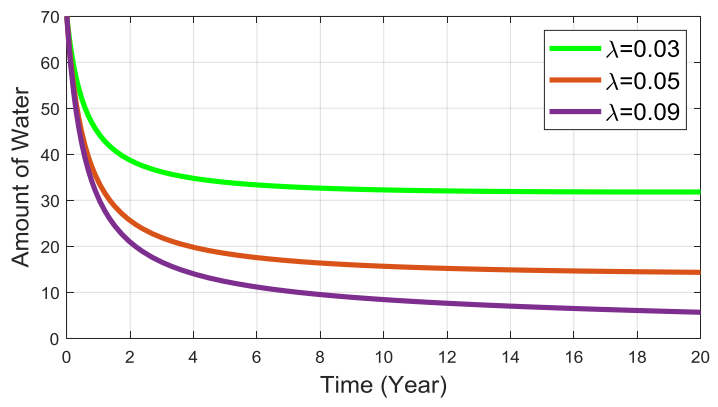

Figure 6. Groundwater level is tremendously decreased due to increase of deforestation rate from $\lambda=0.03$ to $\lambda=0.09$

Figure 6 represents the variation of groundwater level for different values of deforestation rate $\lambda$ with time while evaporation rate, pumping rate and other effects are constant. We observe that groundwater level is decreased extensively due to increase of deforestation rate $\lambda$.

\section{Phase Portrait Diagram}

Now, we have shoe the the dynamical behavior of the model (1)-(3) by plotting phase-plane diagram based on the respective parameters present in the model. In these phase-planes, small arrows in Figures 7-8 show the direction field; the red dot represents the equilibrium point and dashed lines are the nullclines. 


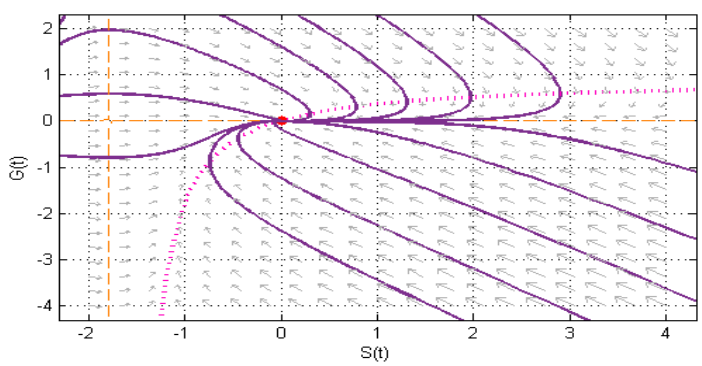

Figure 7. Phase plane and nullclines for the model (1)-(3) with the parameter values $\alpha=.05, \beta=0.3005, \omega=0.06$ and $\lambda=0.030$

\section{Conclusions}

In this paper, a mathematical model on groundwater level declination is presented with qualitative and quantitative analysis. From the numerical simulations, it is clear from Figures 3 and 4 that, the groundwater level is significantly decreased due to increase of pollution rate while the falling of groundwater level is tremendously increased due to lack of storage system. Again, Groundwater and surface water are connected to each other. When groundwater is used superfluously, then lakes, streams and rivers that are connected to subsurface water can also have their supply diminished. We also observe from Figures 5 and 6 that, Groundwater level is tremendously decreased due to increase of deforestation rate, where falling of groundwater level is extensively increased for higher rate of evaporation that is caused of global warming. Lastly, we conclude that the proposed model can be helped for the researchers and planners who are associated with the research of sustainable groundwater management. It also may be helpful for the government to make and take decision regarding the prevention of groundwater level declination as well as may be increase the public awareness in case of using groundwater.

\section{Acknowledgements:}

The first author is greatly acknowledged the partial support of national science and technology (NST) fellowship, under Ministry of Science and Technology, Government of the People's Republic of Bangladesh, bearing ID: MSc-181205, Serial: 708, Merit: 267, No: 39.00.0000.012.002.04.19-06, Session: 2019-2020. The authors also acknowledge the research grant Ref. No.-
39.00.0000.009.06.024.19-12/410(EAS) supported by Ministry of Science and Technology, Government of the People's Republic of Bangladesh. Supports with Ref:: 17-392 RG/MATHS/AS IFR3240297753 funded by TWAS, Italy and Ref. no.6(74) UGC/ST/Physical-17/2017/3169 funded by the UGC, Bangladesh are also acknowledged.

\section{References:}

[1] Attaullah, Sohaib M. Mathematical Modeling and Numerical Simulation of HIV Infection Model. Results in Applied Mathematics 2020; 7: e100118.

[2] Bagherzadeh A, Hoseini AV, Totmaj LH. The Effects of Climate Change on Normalized Difference Vegetation Index (NDVI) in the Northeast of Iran. Model Earth Syst Environ 2020; 6(2): 671-683.

[3] Bangladesh Water Development Board, https://www.hydrology.bwdb.gov.bd/index. php?pagetitle=ground_water_daily_data; 2020 [accessed 13 September 2020].

[4] Bear J, Verruijt A. Modeling Groundwater Flow and Pollution: Theory and Applications of Transport in Porous Media. D Reidel Publishing Company, Holland; 1987.

[5] Biswas MHA, Haque M M, Mallick UK. Optimal Control Strategy for the Immunotherapeutic Treatment of HIV Infection with State Constraint. Optimal Control Applications and Methods 2019; 40(3):1-12.

[6] Biswas MHA, Hossain MR, Mondal MK. Mathematical Modeling Applied to Sustainable Management of Marine Resources. Procedia Engineering 2017; 194: 337-344.

[7] Biswas MHA, Rahman T, Haque N. Modeling the potential impacts of global climate change in Bangladesh: an optimal control approach. J Fundam Appl Sci 2016; 8(1):1-19.

[8] Brammer H, Ravenscroft P. Arsenic in Groundwater: A Threat to Sustainable Agriculture in South and South-east Asia. Environment International 2009; 35(3): 647654.

[9] Brenner S, Coxon G, Nicholas JKH, Freer J, Hartmann A. Process-based Modelling to 
Evaluate Simulated Groundwater Levels and Frequencies in a Chalk Catchment in Southwestern England. Natural Hazards and Earth System Sciences 2018; 18: 445-461.

[10] Brouyere S, Carabin G, Dassargues A. Climate Change Impacts on Groundwater Resources: ModelledDeficits in a Chalky Aquifer, Geer Basin, Belgium. Hydrogeology Journal 2004; 12:123-134.

[11] Buonomo B, Vargas-De-León C. Global stability for an HIV-1 Infection Model Including an Eclipse Stage of Infected Cells. Journal of Mathematical Analysis and Applications 2012; 385: 709-720.

[12] Burges DN, Borrie MS. Modeling with Differential Equations. Ellis Horwood Limited, New York, USA; 1981.

[13] Cheng Y, Wang J, Yang X. On the Global Stability of a Generalized Cholera Epidemiological Model. Journal of Biological Dynamics 2012; 6(2): 1088-1104. DOI:10.1080/17513758.2012.728635.

[14] De Jesus EX, Kaufman C. RouthHurwitz criterion in the examination of eigenvalues of a system of nonlinear ordinary differential equations. Phys Rev A 1987; 35(12):5288.

[15] Hoque MA, Hoque MM, Ahmed KM. Declining groundwater Level and Aquifer Dewatering in Dhaka Metropolitan Area, Bangladesh: Causes and Quantification. Hydrogeology 2007;15(3): 1523-1534.

[16] Islam MA, Imran MBU, Biswas MHA. Modeling the Effects of Transmission Dynamics of Malaria: A Mathematical Approach on Healthcare. Proceedings of the International Conference on Industrial Engineering and Operations Management 2020; Dubai, UAE, 2191-2202.

[17] Khan MJ, Shah BA, Nasir B. Groundwater quality assessment for drinking purpose: a case study from Sindh Industrial Trading Estate, Karachi, Pakistan. Model Earth Syst Environ 2020; 6(1): 263-272

https://doi.org/10.1007/s40808-019-00676$x$.

[18] Khatun MS, Biswas MHA. Modeling the Effect of Adoptive T cell Therapy for the Treatment of Leukemia. Computational and
Mathematical Method 2019. doi: $10.1002 / \mathrm{cmm} 4.1069$.

[19] Murray JD. Mathematical Biology: I. An Introduciton, Third Edition, Springer, New York, USA; 1989.

[20] National Water Policy, Ministry of Water Resources, Government of the People's Republic of Bangladesh, Dhaka, Bangladesh; 1999.

[21] Ross SL. Differential Equations. Third Edition, John Wiley and Sons Inc., New York, USA; 2004.

[22] Vargas C. Global Stability of Infectious Disease Models with Contact Rate as A Function of Prevalence Index. Mathematical Biosciences and Engineering 2017; 14: 1019-1033. doi:10.3934/mbe.2017053.

[23] Weather and Climate Forecast Bangladesh, https:// www.weather atlas.com/en/Bangladesh/Dhaka-climate;

2020

\section{Creative Commons Attribution License 4.0 (Attribution 4.0 International, CC BY 4.0)}

This article is published under the terms of the Creative Commons Attribution License 4.0 https://creativecommons.org/licenses/by/4.0/deed.en_US 